\title{
Principais condutas acerca da gestação normal com doença trofoblástica: uma revisão integrativa de literatura
}

\author{
Main approaches to normal pregnancy with trophoblastic disease: an integrative literature \\ review
}

Principales enfoques para el embarazo normal con enfermedad trofoblástica: una revisión integradora de la literatura

Jacinta Lima Rogério Cardoso ${ }^{1,2 *}$, Bruno Monção Paolino ${ }^{2,4}$, Cintia Cardoso Pinheiro², Larissa Boaes Nascimento ${ }^{1,2}$, July Jenny Blas Rosales ${ }^{2}$, Rebecca Lima Sabba Guimarães Vieira ${ }^{3}$, Giovana Coimbra Luzeiro ${ }^{2,4}$.

\section{RESUMO}

Objetivo: Descrever sobre situações de gestação normal com feto único ou gemelar e Doença trofoblástica (DTG) gestacional coexistente. Métodos: O trabalho foi elaborado a partir revisão bibliográfica, baseado em artigos científicos úteis e pertinentes ao tema proposto. Para localizar as publicações as bases de dados eletrônicas utilizadas foram: SCIELO e PUBMED, os descritores da busca foram: doença trofoblastica gestacional, Gestação gemelar, Mola hidatiforme (MH). Para seleção dos artigos utilizou-se critérios de inclusão e exclusão. Resultados: Estudos já concluídos sobre doença trofoblástica gestacional, resultaram que a investigação é crucial para tratamento correto, além de utilizar-se de exame físico e história clínica para melhor diagnóstico. A ultrassonografia (US) e gonadotrofina coriônica humana ( $\beta$-hCG) devem ser solicitados em todos os casos, o tratamento de DTG é feito com vácuo-aspiração. Considerações finais: Em toda a literatura disponível sobre gestação com feto normal e DTG recomenda a análise genética do cariótipo fetal, para identificar fetos triplóide associados a má formações em mola parcial e que evoluem com óbito ou fetos diplóides associados a mola completa que geralmente são saudáveis e apresentam como gestação normal com DTG do tipo mola completa, podendo evoluir com nascimento viável apesar das possíveis complicações maternas que podem ou não acontecer.

Palavras-chave: Doença trofoblastica gestacional, Gestação gemelar, Mola hidatiforme.

\begin{abstract}
Objective: To describe situations of normal pregnancy with a single or twin fetus and coexisting gestational trophoblastic disease (GDT). Methods: The work was based on a bibliographic review, based on useful scientific articles relevant to the proposed theme. To locate the publications, the electronic databases used were: SCIELO and PUBMED, the search descriptors were: gestational trophoblastic disease, twin pregnancy, hydatidiform mole $(\mathrm{MH})$. For the selection of articles, inclusion and exclusion criteria were used. Results: Studies already concluded on gestational trophoblastic disease, resulted in the investigation being crucial for correct treatment, in addition to using physical examination and clinical history for a better diagnosis. Ultrasonography (US) and human chorionic gonadotropin ( $\beta-h C G)$ must be ordered in all cases, the treatment of DTG is done with vacuum-aspiration. Final considerations: In all available literature on gestation with
\end{abstract}

\footnotetext{
${ }^{1}$ Hospital Universitário Getúlio Vargas (HUGV), Manaus-AM. *E-mail: jacintalimarc@gmail.com

2 Universidade Federal do Amazonas (UFAM), Manaus-AM.

3 Universidade Estadual do Amazonas (UEA), Manaus-AM.

4 Universidade Estadual Paulista (UNESP), São Paulo-SP.
} 
normal fetus and DTG recommends genetic analysis of the fetal karyotype, to identify triploid fetuses associated with partial spring malformations that evolve with death or diploid fetuses associated with full spring that are generally healthy and they present as normal pregnancies with complete spring type GDT, which can evolve with a viable birth despite the possible maternal complications that may or may not happen.

Keywords: Gestational trophoblastic disease, Twin pregnancy, Hydatidiform mole.

\section{RESUMEN}

Objetivo: Describir situaciones de embarazo normal con un feto simple o gemelo y enfermedad trofoblástica gestacional (DTG) coexistente. Métodos: El trabajo se basó en una revisión bibliográfica, basada en artículos científicos útiles relevantes para el tema propuesto. Para localizar las publicaciones, las bases de datos electrónicas utilizadas fueron: SCIELO y PUBMED, los descriptores de búsqueda fueron: enfermedad trofoblástica gestacional, embarazo gemelar, mola hidatidiforme $(\mathrm{MH})$. Para la selección de artículos, se utilizaron criterios de inclusión y exclusión. Resultados: Los estudios ya concluidos sobre la enfermedad trofoblástica gestacional, dieron como resultado que la investigación fuera crucial para el tratamiento correcto, además de utilizar el examen físico y la historia clínica para un mejor diagnóstico. La ecografía (EE. UU.) Y la gonadotropina coriónica humana ( $\beta$-hCG) deben solicitarse en todos los casos, el tratamiento de DTG se realiza con aspiración al vacío. Consideraciones finales: En toda la literatura disponible sobre gestación con feto normal y DTG recomienda el análisis genético del cariotipo fetal, para identificar fetos triploides asociados con malformaciones parciales de primavera que evolucionan con la muerte o fetos diploides asociados con primavera completa que generalmente son saludables. y se presentan como embarazos normales con GDT tipo resorte completo, que puede evolucionar con un parto viable a pesar de las posibles complicaciones maternas que pueden ocurrir o no.

Palabras clave: Enfermedad trofoblástica gestacional, Embarazo gemelar, Mola hidatiforme.

\section{INTRODUÇÃO}

A doença trofoblástica gestacional (DTG) é conhecida com uma doença rara, podendo assim ser conhecida como uma irregularidade proliferativa que alcança as células fazem a composição do tecido trofoblástico placentário, cito e sincicio trofoblasto, mesmos que seus diversos estágios histológicos espacem na propensão para regressão, invasão, metástase e recorrência (SECKL MJ, et al., 2013), ainda segundo o autor apesar de ser mais incidente nas mulheres em idade fértil também podem ser observadas nos extremos reprodutivos como mulheres abaixo de 25 anos e mulheres na menopausa.

A DTG acontece por meio da fertilização anormal e se apresenta especialmente em duas formas principais: mola hidatiforme completa (CHM) e mola hidatidiforme parcial (PHM) O achado comum da DTG é a presença elevada do hormônio gonadotrofina coriônica humana (HCG), marcador biológico da gravidez.

$\mathrm{Na}$ atualidade, a vasta disponibilidade da ultrassonografia (US) e da dosagem do beta-hCG sérico tem causado, maiores diagnósticos precoces da DTG, em muitos casos, antes do surgimento das manifestações clínicas iniciais (BRAGA A, 2019).

A neoplasia trofoblástica gestacional (NTG) é caracterizada por lesões malignas com potencial de invasão local e metástase com antecedente gestacional.

É definida por presença de platô e/ou queda menor que 10\% do beta hCG (BAERGEN RN, DIZON DS, 2019), estão incluídas nesse grupo: mola invasora (MHI), coriocarcinoma (CC), tumor trofoblástico de sítio placentário (TTSP) e tumor trofoblastico epitelióide (TTE).

Braga A (2019), ressalta que a suspeita da gravidez molar tem início quando há sangramento transvaginal em gestação inicial, o que pode dar indícios de ameaça ou aborto consumado, relacionados à presença do beta-hCG elevado no sangue materno. A confirmação da doença se dá quando é realizado exame 
ultrassonográfico, e de forma definitiva com o estudo anatomopatológico de material abortado. Gestação normal, ou seja, com feto sem alterações e vivo, porém, com DTG concomitante é uma situação especial e ainda mais rara ocorrendo cerca de 1 / $20.000-100.000$ gestações.

Nos casos de gestação normal com DTG as principais complicações observadas maternas e fetais são pré eclampsia, sangramento vaginal, hiperemese gravídica, hipertireoidismo ou tempestade tireoidiana, cistos teca luteinicos, síndrome de hiperestimulação ovariana, síndrome de angústia respiratória aguda, embolização trofoblástica, edema agudo de pulmão e tromboembolismo pulmonar assim como óbito fetal, aborto espontâneo e prematuridade (FERRAZ L, 2013).

O desenvolvimento da DTG em população de mulheres com idade de reprodução encontra-se expressivamente em valores maiores em mulheres cuja idade é superior a 35 anos e tendo um discreto aumentado em mulheres com menos de 20 anos, mostrando ter essa doença predileção para os extremos de idade, como a fertilização in vitro (FIV) (BRAGA A, 2019).

Diante dos riscos nesse tipo de gestação muitas vezes sua manutenção é questionada e a decisão de se interromper a mesma em detrimento da preservação da saúde materna torna-se um dilema constante para médicos e pacientes.

Tratando-se de doença rara, grande parte das literaturas disponíveis e atualizadas são relatos de caso. Buscando encontrar mais informações especialmente quanto a conduta nos casos de gestação de feto normal com DTG esse trabalho objetivou realizar a revisão da bibliográfica a fim de averiguar novas possibilidades na condução desta patologia.

\section{MÉTODOS}

O trabalho foi elaborado a partir de uma revisão bibliográfica do tipo integrativa, sendo que para o acesso de estudos básicos o presente artigo foi fundamentado em outros artigos científicos que se mostraram úteis e pertinentes ao tema proposto.

As bases de dados eletrônicas utilizadas para a escolha dos artigos foram: Scientific Eletronic Library online (SCIELO), e US National Library of Medicine National Institutes of Health (PUBMED).

Como forma de localizar os artigos os descritores utilizados para a busca nas bases de dados foram: Doença trofoblástica gestacional, Gestação gemelar, Mola hidatiforme.

Fazendo uso de pressupostos para atingir aos critérios, de inclusão e exclusão, foram utilizados como critérios de inclusão artigos de 2010 a 2019 na língua portuguesa, espanhola e inglesa, com o tema doença trofoblástica, gestação gemelar, e para os critérios de exclusão: desconsiderou artigos que não adequavam o tema no seu contexto na íntegra, sendo somente 15 selecionados pois os mesmos apresentaram o objetivo do trabalho.

\section{RESULTADOS}

Relacionando os artigos do presente estudo verificou-se por meio do Quadro 1, os resultados e conclusões das pesquisas, referente a pacientes que tiveram uma gestação normal com doença troflobástica. 
Quadro 1 - Principais resultados dos achados relacionados gestação normal com doença trofoblástica, seus resultados e conclusões.

\begin{tabular}{|c|c|c|c|c|c|}
\hline & Autor e Ano & Revista & Qualis & Título & Resultados/Conclusão \\
\hline 1 & $\begin{array}{l}\text { Oliveira AS et } \\
\text { al. } \\
(2013)\end{array}$ & $\begin{array}{l}\text { Reproduçã } \\
\text { o e } \\
\text { climatério }\end{array}$ & B1 & $\begin{array}{l}\text { Gestação gemelar com } \\
\text { mola hidatiforme } \\
\text { completa e feto vivo } \\
\text { após ovodoação e } \\
\text { fertilização } \\
\text { em vitro: relato de } \\
\text { caso. }\end{array}$ & $\begin{array}{l}\text { Neste estudo a paciente optou por manter uma gestação, sendo que, o parto foi } \\
\text { realizado com } 29 \text { semanas, sem complicações maternas. O recém-nascido evoluiu } \\
\text { com bom desenvolvimento neuropsicomotor, e sem sequelas. }\end{array}$ \\
\hline 2 & $\begin{array}{l}\text { Gupta K, et al } \\
(2015)\end{array}$ & WMJ & - & $\begin{array}{l}\text { Detecção Precoce por } \\
\text { ultra-som de Parcial } \\
\text { mola Hidatiforme } \\
\text { Parcial Com feto vivo } \\
\text { coexistente. }\end{array}$ & $\begin{array}{l}\text { Neste estudou foi enfatizado o papel da ultra-sonografia no diagnóstico desta condição } \\
\text { no início da gravidez, conseguindo ter uma visão sucinta dos mecanismos etiológicos, } \\
\text { sua possíveis complicações e manejo clínico é fornecido. Foi descrita como uma } \\
\text { ferramenta de diagnóstico eficaz para diagnosticar mola hidatiforme com coexistente } \\
\text { feto vivo, onde o seu diagnóstico precoce é importante para a estratificação de risco e } \\
\text { assim facilitar uma decisão pelo paciente de interromper a gravidez ou continuar até } \\
\text { a termo com acompanhamento de perto após o parto. }\end{array}$ \\
\hline 3 & $\begin{array}{l}\text { Cristina } \\
\text { Flórez F,et al. } \\
(2016)\end{array}$ & $\begin{array}{l}\text { Chil Obstet } \\
\text { Gynecol }\end{array}$ & - & $\begin{array}{l}\text { Gravidez molar vivo } 25 \\
\text { semanas fetal. relatório } \\
\text { da autópsia. }\end{array}$ & $\begin{array}{l}\text { O estudo histopatológico da placenta e o produto de gestação permitir um diagnóstico } \\
\text { definitivo para determinar a monitorização do paciente e, assim, reduzir complicações. }\end{array}$ \\
\hline 4 & $\begin{array}{l}\text { Maeda Y, et } \\
\text { al. (2018) }\end{array}$ & $\begin{array}{l}\text { J. Obstet. } \\
\text { Gynaecol }\end{array}$ & B1 & $\begin{array}{l}\text { Coriocarcinoma com } \\
\text { múltiplas metástases } \\
\text { pulmonares de mola } \\
\text { hidatidiforme completa } \\
\text { com feto coexistente } \\
\text { durante a gravidez. }\end{array}$ & $\begin{array}{l}\text { A mola hidatiforme completa com feto (MHCF) pode resultar em neoplasia } \\
\text { trofoblástica gestacional NTG de alto risco durante a gravidez. Para um GTN suspeita, } \\
\text { o diagnóstico } \\
\text { A pontuação de Federação Internacional de Ginecologia e Obstetrícia (FIGO) deve } \\
\text { determinar a estratégia de tratamento. Se pacientes com MHCF desejarem continuar } \\
\text { com gravidez, acompanhamento cuidadoso, incluindo radiografia de tórax e ultra- } \\
\text { sonografia, se justifica. }\end{array}$ \\
\hline
\end{tabular}

REAS/EJCH |Vol.12(3) | e3087 | DOI: https://doi.org/10.25248/reas.e3087.2020 Página 4 de $\mathbf{1 2}$ 


\begin{tabular}{|c|c|c|c|c|c|}
\hline 5 & $\begin{array}{l}\text { Chen CP, et } \\
\text { al., (2014) }\end{array}$ & $\begin{array}{l}\text { Taiwan } \\
\text { Journal of } \\
\text { Obstetrics } \\
\& \\
\text { Gynecology }\end{array}$ & B1 & $\begin{array}{l}\text { Diagnóstico molecular } \\
\text { no primeiro trimestre } \\
\text { de mola hidatidiforme } \\
\text { completo associado } \\
\text { com a } \\
\text { gravidez duplo } \\
\text { dizigóticos concebidos } \\
\text { por inseminação intra- } \\
\text { uterina. }\end{array}$ & $\begin{array}{l}\text { Os resultados da análise do marcador polimórfico de reação em cadeia da polymerase } \\
\text { (PCR) identificador de STR mostraram concepção androgênica na massa cística } \\
\text { complexa e concepção biparental no feto vivo. A análise patológica da massa cística } \\
\text { confirmou o diagnóstico de mola hidatiforme completa CHM. Os resultados do Reação } \\
\text { em cadeia da polimerase fluorescente quantitativa (QF-PCR), mostraram herança } \\
\text { biparental no feto normal e homozigose paterna completa no CHM do feto anormal em } \\
\text { todos os STRs, indicando gêmeos dizigóticos e CHM de monospermia. }\end{array}$ \\
\hline 6 & $\begin{array}{l}\text { Garcia M et } \\
\text { al., (2016). }\end{array}$ & $\begin{array}{l}\text { Associação } \\
\text { Médica } \\
\text { Brasileira }\end{array}$ & B2 & $\begin{array}{lr}\text { Resultados } & \text { da } \\
\text { gravidez } & \text { após } \\
\text { quimioterapia } & \text { para } \\
\text { neoplasia trofoblástica. }\end{array}$ & $\begin{array}{l}\text { Não apresentou evidência de diminuição da fertilidade após a quimioterapia para } \\
\text { neoplasia trofoblásticas. As taxas de aborto em pacientes que conceberam dentro de } \\
6 \text { meses após a quimioterapia foram elevadas em relação com os que esperaram mais } \\
\text { tempo. Mostrou-se o aumento de anomalias congénitas. Gestações após a } \\
\text { quimioterapia para neoplasia trofoblástica deve obter uma vigilância clínica devido a } \\
\text { taxas mais elevadas de algumas complicações na gravidez. }\end{array}$ \\
\hline 7 & $\begin{array}{l}\text { Yela DA, et } \\
\text { al. }(2011)\end{array}$ & $\begin{array}{l}\text { Jornal } \\
\text { Brasileiro } \\
\text { de } \\
\text { Patologia e } \\
\text { Medicina } \\
\text { Laboratorial }\end{array}$ & B4 & $\begin{array}{ll}\text { Gestação } & \text { gemelar de } \\
\text { mola } & \text { hidatiforme } \\
\text { completa } & \text { com feto } \\
\text { vivo. } & \end{array}$ & $\begin{array}{l}\text { Concluiu-se que, na suspeita de gestação gemelar molar com presença de feto vivo, } \\
\text { o primeiro passo deve ser a determinação do cariótipo. Deve expor a possibilidade de } \\
\text { a gestação e complicações maternas durante a gestação e de ocorrer transaminase } \\
\text { glutâmico-pirúvica (TGP). A decisão de levar a gestação até o termo dependerá da } \\
\text { equipe médica e dos pais. }\end{array}$ \\
\hline 8 & $\begin{array}{l}\text { Wagner SS, } \\
\text { et al (2008) }\end{array}$ & $\begin{array}{l}\mathrm{J} \\
\text { Ultrasound } \\
\text { Med }\end{array}$ & B2 & $\begin{array}{l}\text { Metastática } \\
\text { gestacional doença } \\
\text { trofoblástica após uma } \\
\text { completar mola } \\
\text { Hidatiforme mistura } \\
\text { com um Anencefálico } \\
\text { feto diagnosticado em } \\
\text { uma gestação de } 10 \\
\text { semanas. }\end{array}$ & $\begin{array}{l}\text { Na maioria dos relatos de moles completos coexistente com feto vivo, o feto vivo é } \\
\text { estrutural e análise do cariótipo normal. Foi relatado os achados ultrassonográficos da } \\
\text { doença trofoblástica gestacional metastática após uma gestação gemelar com uma } \\
\text { toupeira completa e um feto anencéfalo ao vivo. }\end{array}$ \\
\hline
\end{tabular}

REAS/EJCH |Vol.12(3) | e3087 | DOI: https://doi.org/10.25248/reas.e3087.2020 Página 5 de 12 


\begin{tabular}{|c|c|c|c|c|c|}
\hline 9 & $\begin{array}{l}\text { Soares LR, et } \\
\text { al }(2014)\end{array}$ & $\begin{array}{l}\text { Reproduçã } \\
0 \quad \text { e } \\
\text { climatério }\end{array}$ & B1 & $\begin{array}{l}\text { Gestação gemelar com } \\
\text { mola hidatiforme } \\
\text { completa e feto vivo. }\end{array}$ & $\begin{array}{l}\text { Descreveu a evolução clínica de caso de GGMC que evoluiu para crise tireotóxica, } \\
\text { pré-eclâmpsia, interrupcão da Gestação e necessidade de cuidados Intensivos } \\
\text { maternos. Reaizou-se a necropsia fetal evidenciando feto sexo feminino, sem } \\
\text { apresentar malformacões com alteracões placentárias que favorecem } \\
\text { cromossomopatia. Feita a avaliação dos restos ovulares evidenciou vilosidades com } \\
\text { hiperplasia fazer trofoblasto e vesículas,achados compatíveis com mola hidatiforme } \\
\text { completa. }\end{array}$ \\
\hline 10 & $\begin{array}{l}\text { Maestá I, et } \\
\text { al., (1998) }\end{array}$ & RBGO & B4 & $\begin{array}{l}\text { Mola Completa em } \\
\text { Gravidez Gemelar: } \\
\text { Relato de Caso. }\end{array}$ & $\begin{array}{l}\text { Caso de diagnóstico tardio em decorrência da presença de feto relizado no momento } \\
\text { da resolução da gestação e confirmado por estudo histopatológico e citometría de } \\
\text { fluxo. Resolução da Gestação feita por vía transpélvica em decorrência de hemorragia } \\
\text { uterina maciça. Para o seguimento pós-molar evidenciou persistência de níveis } \\
\text { elevados de hormônio gonadotrofina coriônica humana (bhCG), obtendo-se remissão } \\
\text { completa da doença com o Uso do metotrexato. }\end{array}$ \\
\hline 11 & $\begin{array}{l}\text { Ferraz TJSM, } \\
\text { et al., (2013) }\end{array}$ & RBGO & B4 & $\begin{array}{l}\text { Mola hidatiforme } \\
\text { completa em Gravidez } \\
\text { bicoriônica após } \\
\text { Injeção } \\
\text { intracitoplasmática de } \\
\text { espermatozóides. }\end{array}$ & $\begin{array}{l}\text { Ecografia pré-natal realizada na } 12^{\underline{a}} \text { semana de gestação,foi identificado um embrião } \\
\text { morfologicamente normal e uma placenta com características molares. A situação } \\
\text { resultou na terminação eletiva da gravidez. A autopsia e estudo histológico permitiu o } \\
\text { diagnóstico definitivo de uma mola hidatiforme completa coexistindo com feto normal. } \\
\text { A Análise genética mostrou feto diplóide com genoma biparental e Tecido molar com } \\
\text { diploidia paterna. O caso ressaltou como gestações com mola hidatiforme completa } \\
\text { poderão ainda ocorrer, mesmo que a gravidez seja realizada após uma Injeção } \\
\text { intracitoplasmática de espermatozoides. }\end{array}$ \\
\hline
\end{tabular}

REAS/EJCH |Vol.12(3) | e3087 | DOI: https://doi.org/10.25248/reas.e3087.2020 Página 6 de 12 


\begin{tabular}{|c|c|c|c|c|c|}
\hline 12 & $\begin{array}{l}\text { Nobuhara I, } \\
\text { et al., (2018) }\end{array}$ & $\begin{array}{l}\text { Journal of } \\
\text { obstetrics \& } \\
\text { Gynecology }\end{array}$ & B1 & $\begin{array}{l}\text { Doença gestacional } \\
\text { metastática múltipla } \\
\text { trofoblástica depois de } \\
\text { uma gestação gemelar } \\
\text { com mola }\end{array}$ & $\begin{array}{l}\text { Mulher primigesta de } 42 \text { anos, com gravidez anormal (isto é, MHCF) por FIV, } \\
\text { persistindo } \\
\text { Sangramento vaginal grave, levando à interrupção da gravidez com } 10 \text { semanas. Os } \\
\text { exames histopatológicos revelaram que era } \\
\text { Um caso de MHCF. Casos de MHCF podem ter maior potencial maligno e maior risco } \\
\text { de desenvolvimento de GTD e tornam-se mais agressivo biologicamente. O curso } \\
\text { clínico da MHCF após Tecnologia Reprodução Assistida (TRA) parece ser quase a } \\
\text { mesma que sem TRA com base nos resultados da revisão da literatura. }\end{array}$ \\
\hline 13 & $\begin{array}{l}\text { Suksai M, et } \\
\text { al., (2017) }\end{array}$ & $\begin{array}{l}\text { E J of } \\
\text { Obstetrics } \\
\& \\
\text { Gynecology } \\
\text { and } \\
\text { Reproducti } \\
\text { ve Biology }\end{array}$ & B1 & $\begin{array}{lr}\text { Mola } & \text { hidatiforme } \\
\text { completa } & \text { com } \\
\text { coexistência: } & \text { pré- } \\
\text { editores de vida } & \\
\text { nascimento } & \end{array}$ & $\begin{array}{l}\text { Foram identificados } 89 \text { relatos de casos e uma série de } 204 \text { gestantes. As gestações } \\
\text { deram à luz } 78 \text { nascidos vivos ( } 37,86 \%) \text {. Para sintomas clínicos, mulheres grávidas } \\
\text { com pré-natal complicações, incluindo hipertensão induzida pela gravidez (PIH), } \\
\text { hipertireoidismo (HTD) e hiperêmese gravídico }(H G) \text {, desenvolveu eventos perinatais } \\
\text { adversos signi fi cativamente. O baixo nível sanguíneo de hCG foi omelhor preditor de } \\
\text { sobrevida fetal ( } P=0,006)\end{array}$ \\
\hline 14 & $\begin{array}{l}\text { Zilberman } \\
\text { SN, (2019) }\end{array}$ & & - & $\begin{array}{l}\dot{E} \text { uma r mola } \\
\text { hidatiforme completa e } \\
\text { um feto normal } \\
\text { coexistente um efeito } \\
\text { iatrogênico }\end{array}$ & $\begin{array}{l}\text { Nenhuma das três gestações apresentadas neste artigo resultou em um feto viável, } \\
\text { todas foram abortadas. Um dos três pacientes necessitou de quimioterapia devido a } \\
\text { neoplasia trofoblástica gestacional (GTN). }\end{array}$ \\
\hline 15 & $\begin{array}{l}\text { Lin LH, et al., } \\
(2017)\end{array}$ & $\begin{array}{l}\text { Gynecologi } \\
\text { c Oncology }\end{array}$ & $\mathrm{A} 1$ & $\begin{array}{l}\text { Gestações múltiplas } \\
\text { com feto normal } \\
\text { completo e coexistente } \\
\text { na América do } \\
\text { do Sorte e } \\
\text { retrospectiva } \\
\text { multicêntrica e } \\
\text { revisão da literatura }\end{array}$ & $\begin{array}{l}\text { A principal diferença encontrada foi a apresentação do MHCF que está relacionada a } \\
\text { uma maior taxa de risco potencial de vida condições na América do Sul, } 60 \% \text { dos } \\
\text { MHCF administrados com expectativa tiveram bebê viável, e a taxa geral de GTN } \\
\text { neste estudo foi de } 46 \% \text {. A interrupção eletiva da gravidez não influenciou a risco para } \\
\text { GTN; no entanto, a necessidade de término devido a complicações e níveis mais altos } \\
\text { de hCG foram associados com desenvolvimento de GTN no MHCF. }\end{array}$ \\
\hline
\end{tabular}

Fonte: Cardoso JLR, et al., 2020.

REAS/EJCH |Vol.12(3) | e3087 | DOI: https://doi.org/10.25248/reas.e3087.2020 Página 7 de 12 


\section{DISCUSSÃO}

A associação de mola hidatiforme completa e Gestação gemelar é bem incomum, uma vez que na literatura consta em média 200 casos descritos (CHESNAIS AL, et al., 2011). Com o aumento de problemas diversos relacionados à fertilidade é possível observar o crescente número de pessoas que vem utilizando as técnicas de reprodução assistida. Como fertilização em vitro (FIV) e injeção intracitoplasmática de espermatozóides (IICE).

Apesar da grande contribuição aos casos de infertilidade a reprodução assistida, ainda assim, traz um aumento no risco de fertilização de óvulos anormais e dentre outros problemas são observadas gestações de fetos normais com DTG. A FIV pode causar uma fertilização multiespermática e aumentar o risco de DTG proveniente de gestação dispérmica, em contrapartida, a IICE pode evitar a fertilização multiespérmica.

Uma situação encontrada nestes casos é o desejo de permanência da gestação (OLIVEIRA AS, et al., 2013). Cerca de $50 \%$ das pacientes tem o útero expandido para a idade gestacional, que abaixa após o sangramento e volta a crescer por meio da proliferação do trofoblasto remanescente intrauterino (BELFORT P e BRAGA A, 2004).

O feto de uma mola parcial triplóides tende a morrer no primeiro trimestre, enquanto um molar completo com um feto normal permite- se pensar na possibilidade de uma gestação com bom desfecho apesar dos riscos de complicações maternas e fetais. Alguns casos de mola hidatiforme completa com feto (MHCF) após a injeção intracitoplasmática de espermatozóide (IICE) foram relatados, principalmente na população asiática (CHESNAIS AL, 2011).

Ao se observar uma placenta com uma degeneração molar e um feto vivo, deve-se considerar algumas hipóteses: mola parcial com feto triploide e gestação gemelar com feto normal, e mola completa (GGMC). No estudo abordado por Yela DA, et al. (2011), em seu estudo teve com estudo uma paciente Primigesta, 20 anos de com gestação de 17 semanas onde apresentou valores de beta da gonadotrofina coriónica humana ( $\beta$ HCG) inicialmente de $178,510 \mathrm{mUl} / \mathrm{mol}$, marcados pelo exame de ecografia feto vivo com biometria fetal combinando as semanas de gestação, com batimentos cardíacos e movimentos fetais presentes.

O diagnóstico ultrassonográfico recomendou gestação gemelar com feto de 16/17 semanas e mola hidatiforme completa, onde permanecia o plano de clivagem afastando uma placenta normal da mola. Esse acompanhamento por meio da ultrassonografia conseguiu mostrar o crescimento fetal adequado e áreas císticas à borda placentária sem Invasão miometrial. O parto se deu em 40 semanas, por cesárea, devido à distocia funcional.

Comparando o estudo de Yela DA, et al., (2011), e diversas literaturas em relação a qual conduta ser realizada nesse caso pode-se esclarecer que é um caso incomum, há visto que houve a finalização da gestação com feto vivo, não tendo quaisquer intercorrências de complicações maternas e Fetais. Suspeita de Gestação gemelar molar com presença de feto vivo. É imprescindível determinar o cariótipo, e sempre expor aos pais a possibilidade de uma gestação culminar em parto, com complicações maternas e fetais graves.

Em caso de complicações de gravidez molar com feto vivo é preciso expor as condições clínicas e complexas da gravidez uma vez que existe o desejo pelo filho. É válido ressaltar que a interrupção da gravidez é geralmente indicada quando há existência de um feto severamente malformado (LURAIN JR, 2010). Alguns relatos mostram que devido ao alto risco de complicações para a mãe durante o período gestacional em grande maioria dos casos a evolução do processo é a interrupção da Gestação (SECKL MJ, et al., 2010).

O código penal brasileiro deixa claro as condições em que não se pune o médico por pratica de aborto nos termos do artigo 128 nos seus incisos I e II nos casos de aborto necessário (terapêutico) quando não há outra forma de salvar a vida da gestante ou aborto sentimental (humanitário) em caso de estupro. Ainda segundo o artigo 128 no seu inciso I o aborto terapêutico é considerado legal nos casos em que o mesmo é a única forma e não a melhor para salvaguardar a vida da gestante (GRECO R, 2017).

Segundo o doutrinador em direito Greco R, (2017), em ambos os casos acima citados cabe ao magistrado quando solicitado assumir a responsabilidade de fazer valer a lei em benefício da mulher protegendo o médico da punibilidade. $O$ autor citado relata ainda que não se faz necessária autorização previa para realização de aborto terapêutico nos casos de urgência em que haja risco de morte materna. 
O artigo 24 do código penal do Brasil nos termos da lei define estado de necessidade quem pratica o fato para salvar de perigo atual (CÓDIGO PENAL COMENTADO, 2017). Pelo fato da utilização da palavra "atual" no código penal, falar sobre aborto terapêutico, pode ocorrer insegurança e questionamento sobre a interpretação dos artigos 128 e 24 quanto a possibilidade de se oferecer eletivamente, como ocorre em países onde o aborto não é criminalizado.

Um estudo de caso abrangendo 77 gestações gemelares com mola hidatiforme, onde o estudo verificou uma porcentagem equivalente a $40 \%$ dos recém-nascidos foram normais sem aumentar risco de complicações maternas.

Outro achado é que se as complicações forem diagnosticadas e tratadas precocemente, o risco de maliguinizacão da mola hidatiforme não aumentou com a idade gestacional (SEBIRE NJ, et al, 2002).

Gravidez gemelar com doença trofoblástica gestacional (DTG) do tipo mola hidatiforme completa e feto coexistente normal é rara e associada a complicações graves durante a gravidez podendo evoluir para neoplasia trofoblástica gestacional (NTG) (NOBUHARA I, et al., 2018). Ainda segundo o mesmo autor apenas uma dúzia de casos de MHCF após tecnologias de reprodução assistida (TRA) ou fertilização in vitro (FIV), foram relatados até 2018.

É importante lembrar que nos casos de aborto terapêutico por complicações que levam ao risco de morte materna antes do esvaziamento uterino a paciente deve ser estabilizada e avaliada para outras possíveis situações de risco como crise tireotóxica, anemia, insuficiência renal dentre outras, sendo prudente avaliação clínica e laboratorial.

De acordo com Gupta K, (2015), as principais possibilidades de uma gestação com feto e mola coexistente são (1) gestação molar completa com um feto normal diploide coexistente dizigóticos, (2) Mola hidatiforme parcial com feto diplóide e feto normal coexistente dizigóticos e (3) mola hidatiforme parcial com feto anormal triploide coexistente em uma gravidez de gêmeos monozigóticos.

Uma complicação de grande risco fatal de gravidezes molares é o risco de doença trofoblástica gestacional persistente (DTGp) (PIURA, B, et al., 2008). A incidência de DTGp após esvaziamento cirúrgico/uterino em hidatiforme completa com feto é respeitosamente superior, quando comparada a mola hidatidiforme parcial com feto (1,028\% vs 3-5\%) (BRUNER DI; PRITCHARD AM, J,2013).

Após fazer o esvaziamento do útero inicia-se a seqüência pós-molar, que objetiva monitorar a curva de hCG a fim de conseguir constatar a evolução para NTG. A molécula de hCG é complexa e existe assim variados testes para sua dosagem, ainda que nenhum deles ainda tenha sido feito especificamente para 0 seguimento da DTG, tão somente para o diagnóstico de gravidez (COLE LA, 2009).

Fatores de risco etiológicos para molas parciais são acometidos por abortos, ciclos irregulares, e idade materna. Segundo Juárez AA, et al., (2010), a presença da mola parcial com feto vivo é anormal para a variedade de complicações clínicas materno-fetal que podem surgir, adjuntos com a proliferação de trofoblasto, tais como o desenvolvimento da pré-eclâmpsia precoce, hemorragia do hipertiroidismo materna primeiro trimestre entre outro.

Garcia M et al., (2016), em seu estudo comparou que as grávidas que foram concebidas no prazo de 6 meses após o fim da quimioterapia buscando avaliar as condições futuras de reprodução para neoplasia trofoblástica gestacional, conclui que a introdução de quimioterapia alterou o prognóstico dos doentes com neoplasia trofoblástica gestacional. Apesar da preocupação quanto a fertilidade, os estudos não mostraram diminuição na mesma.

Resultado importante foi encontrado por Matsui $\mathrm{H}$, et al, (2004), onde foi concluído em seu estudo que a incidência de aborto, morte fetal e mola foi elevada quando relacionado as mães que tiveram gestação dentro de 6 meses após a conclusão da quimioterapia em comparação com aqueles que esperou 1 ano.

É importante que os ciclos de quimioterapia sejam realizados até que haja a normalização do hCG., sendo que se faça necessário poupar atrasos nos intervalos de tempo entre as sessões de quimioterapia sem uma causa específica, pelo risco de ampliar a doença resistente (LURAIN JR, 2008). 
Aconselha-se as pacientes terem um controle da gestação para pelo menos 1 ano após a quimioterapia com níveis de hCG negativas, a fim de conseguir ter uma garantia de se tornar uma gravidez segura, não deixando de ressaltar que este é um período crítico que pode desencadear a maioria dos recaídas. A taxa superior de aborto nos primeiros 6 meses após o final do tratamento é mostrada pela literatura e assim destaca-se a importância de não engravidar antes desse período (BRAGA U, et al., 2009).

Após obter a normalização do hCG, recomenda-se que se faça a quimioterapia de consolidação sendo realizado em média dois ou três ciclos, especialmente para NTG de alto risco, buscando evitar recidivas (GOLDSTEIN DP e BERKOWTIZ RS, 2012).

Nos casos de gestação gemelar com mola completa (GGMC) Segundo Soares LR (2014), é indicado o esvaziamento da cavidade uterina após resolução da gestação e apesar da preferência por vacuoaspiracão (AMIU), que apresenta menor taxa de perfuração uterina sabe-se que o material obtido pela curetagem apresenta menor índice de autólise (ANDRADE JM, 2009), e seria mais adequado para uma histopatologia, porém o risco aumentado de hemorragia e perfuração uterina torna o AMIU o método de eleição e o mais usado.

Além disso o seguimento por meio da Dosagem sérica de beta hCG é obrigatório e a radiografia de tórax assim como o exame físico da vagina devem ser realizados, visto que pulmões e vagina são os sítios mais comuns de metástase nos casos que evoluem para NTG.

Apesar de alguns estudos apontarem para uma associação entre mola completa e feto normal pós tratamentos de fertilidade, poucos são os dados clínicos para confirmar essa hipótese. Dessa forma o maior risco de gestação gemelar com mola completa após tecnologia de reprodução assistida ainda permanece incerto (MASSARDIER J, et al.,2009).

Fazer a diferenciação diagnostica entre mola hidatiforme completa (MHC) e mola hidatiforme parcial (MHP) é fundamental ao se pensar em DTG visto que a MHC apresenta um risco considerável maior para Neoplasia trofoblástica gestacional (NTG) e de complicações graves maternas e fetais ao passo que o risco da MHP progredir para NTG é inferior a 5\% (ZILBERMAN SN, 2019).

Maestá I, et al (1998), em sua abordagem relata que a conduta da gestação gemelar com mola completa e feto normal irá depender de individualidade de cada caso, sendo em alguns utilizado a conduta conservadora.

Quando se faz o diagnóstico antecipado da $\mathrm{MH}$, sendo caracterizado na décima primeira podendo elevar atá a décima segunda, consegue amenizar a ocorrência de complicações clínicas dentre elas como préeclâmpsia, hipertireoidismo, anemia, hiperemese e síndrome do desconforto respiratório (SDR) (MAESTÁ I, 2012).

De acordo com Zilberman SN (2019), mulheres com gestação com feto normal e DTG coexistente escolhem o aborto em 10-62\% dos casos e que essa grande variação estatística pode ser atribuída as diferenças de origens étnicas, religiosas, idade materna e estado civil. E relata ainda em seu estudo que mulheres que optaram pela manutenção da gestação $25 \%$ a $50 \%$ terminaram com aborto espontâneo ou morte fetal intrauterina, geralmente $\leq 24$ semanas de idade gestacional, ou seja, fetos com baixas chances de viabilidade.

Em contrapartida estudos apontam que pacientes que tiveram remissão natural da $\mathrm{MH}$ apresentam 99\% de chance de ter uma gravidez normal, existindo assim apenas $1 \%$ de risco de nova MH (GARNER EI, et al, 2007). Já Luriana JR, (2010), em sua colocação perante alguns estudos conseguiu evidenciar que $40 \%$ dessas gestações resultam no final em feto viável e normal.

Outra dificuldade encontrada pelas pacientes com DTG é terem que lidar com o desconhecido durante o período gestacional com o estresse de em muitos casos ocorrer a perda gestacional, em alguns casos precisar fazer o tratamento com o auxílio da quimioterapia e sempre tendo como esperança alcançar os índices normais do hCG (FERREIRA EG, et al., 2009). O medo dessa doença ainda não muito conhecida, de ter que passar por tratamento e não ter garantias para uma gravidez futura são condições que modificam toda a rotina de vida da mulher (MAESTÁ I, 2012). 
É preciso que os profissionais estejam capacitados e especializados quanto a essa patologia, fim de conseguir proporcionar a garantia das mulheres que encontram-se nas condições de ter uma gestação normal com doença trofoblástica, de maneira que seja feita um período de conscientização ressaltando sobre a importância de medidas preventivas e recuperativas que visam oferecer maior qualidade de vida, entendendo a sua magnitude e assim alcançar o conhecimento quanto a essa patologia, bem como suas classificações, agravos, evoluções e tratamento, de modo a minimizar riscos de neoplasia e metástase, efetivando o diagnóstico antecipadamente e estabelecer o tratamento que seja mais adequado (SOARES MKP, et al, 2017).

\section{CONSIDERAÇÕES FINAIS}

Atualmente se recomenda conduta expectante desde que os genitores desejem a manutenção da gestação após toda a explicação das possíveis complicações clinicas que possam ocorrer ou a interrupção da mesma por aborto terapêutico em caso de risco de morte materna pelos mesmos motivos observados na literatura. A suspeita deve ser precoce em casos de gestação normal com DTG em situações de US sugestivo, BHCG quantitativo elevado além do esperado, história prévia de DTG, idade materna avançada, uso de TRA e história familiar de DTG. É consenso ainda realizar acompanhamento pré-natal individualizado, rigoroso e com menor intervalo entre as consultas voltado especialmente para a prevenção sendo assim, possível identificação precoce de complicações clinicas e, portanto, deve sempre se atentar para a realização de um segmento obrigatório com dosagem de BHCG quantitativo após o esvaziamento uterino e por fim dispor de orientações sobre planejamento e futuro reprodutivo da paciente.

\section{REFERÊNCIAS}

1. ANDRADE JM. Mole hidatiforme e Doença trofoblástica gestacional. Rev Bras Ginecol Obstet 2009; 31: 94-101.

2. BAERGEN RN, DIZON DS. Gestational trophoblastic disease: Pathology. UpToDate, Waltham, MA, 2019.

3. BELFORT P, BRAGA A. The changing clinical presentation of molar pregnancy. Rev Bras Ginecol Obstet. 2004;26(6):483-8.

4. BRAGA A, et al. Doença trofoblástica gestacional. FEMINA 2019;47(1): 6-17

5. BRAGA U, et al. Materna e resultados perinatais da primeira gravidez após a quimioterapia para trofoblástica gestacional neoplasia em mulheres brasileiras. Gynecol Oncol. 2009; 112 (3): 568-71.

6. BRUNER DI, PRITCHARD AMJC. Ruptura uterina devido à invasivo metastático trofoblástica gestacional neoplasia. Oeste J Emerg Med. 2013; 14 (5): 444-447.

7. CHEN CP, et al. First-trimester molecular diagnosis of complete hydatidiform mole associated with dizygotic twin pregnancy conceived by intrauterine insemination. Taiwanese Journal of Obstetrics \& Gynecology 53.2014; 572-578.

8. CHESNAIS AL, et al. Gravidez gemelar com dois completa hydatiform toupeira e coexistente feto vivo: relato de um não pré-natal caso diagnosticado. Annales de pathologie. 2011; 31 (4): 299-302.

9. COLE LA. New discoveries on the biology and detection of human chorionic gonadotropin. Reprod Biol Endocrinol. 2009;7(1):8

10. CRISTINA FLÓREZ F. et al.Gravidez molar vivo 25 semanas fetal. Relatório da autópsia. Rev. chil. obstet. ginecol. 2016;81; 6 .

11. FERRAZ TJSM, et al. Mola hidatiforme completa em Gravidez bicoriônica após Injeção intracitoplasmática de espermatozóides. Rev Bras Ginecol Obstet. 2013; 35 (1): 39-43.

12. FERREIRA EG, et al. Assessment of quality of life and psychologic aspects in patients with gestational trophoblastic disease. J Reprod Med. 2009;54(4):239-44.

13. GARCIA MT, et al. Pregnancy outcomes after chemotherapy for trophoblastic neoplasia. Revista Da Associação Médica Brasileira, 2016;62(9), 837-842.

14. GARNER EI, et al. Gestational trophoblastic disease. Clin Obstet Gynecol. 2007; 50:112-22.

15. GOLDSTEIN DP, BERKOWTIZ RS. Current management of gestational trophoblastic neoplasia. Hematol Oncol Clin North Am. 2012; 26(1):111-31

16. GRECO R. Código Penal comentado.11. ed. Niterói, RJ. Impetus, 2017.

17. GUPTA K, et al. Detecção Precoce por ultra-som de Parcial mola Hidatiforme Parcial Com feto vivo coexistente. Outubro 2015, WMJ.

18. JUÁREZ AA. Feto vivo hidatiforme parcialmente com o segundo trimestre. Rev Chil Obstet Gynecol $2010 ; 75$ (2): 137 9.

19. LIN LH, et al., Multiple pregnancies with complete mole and coexisting normal fetus in North and South America: $A$ retrospective multicenter cohort and literature review.Gynecol Oncol. 2017 Apr;145(1):88-95.

20. LURAIN JR. Doença trofoblástica gestacional: epidemiologia, patologia, apresentação clínica e diagnóstico de doença 
trofoblástica gestacional, e gestão de mola hidatiforme. Am J Obstet Gynecol. 2010; 203 (6): 531-539.

21. LURAIN JR. Gestational trophoblastic disease II: classification and management of gestational trophoblastic neoplasia. Am J Obstet Gynecol. 2011;204(1):11-8.

22. LURIANA, JR. Doença trofoblástica gestacional I: epidemiologia, patologia, apresentação clínica e diagnóstico de doença trofoblástica gestacional e gestão de mola hidatiforme. Am J Obstet Gynecol,p. 1-9 de 2010.

23. MAEDA Y, et al., Coriocarcinoma com múltiplas metástases pulmonares de mola hidatidiforme completa com feto coexistente durante a gravidez. J. Obstet. Gynaecol. Res. 2018.

24. MAESTA I, BRAGA A. Desafios do tratamento de pacientes com doença trofoblástica gestacional. Rev. Bras. Ginecol. Obstet. vol.34 no.4 Rio de Janeiro Apr. 2012.

25. MAESTÁ I, et al. Mola Completa em Gravidez Gemelar: Relato de Caso. RBGO 20(7): 415-419, 1998.

26. MASSARDIER J, et al. gravidez de gêmeos com mola hidatiforme completa e feto coexistente. Resultados obstétricos e oncológicas em uma série de 14 casos. Eur J Obstet Gynecol Reprod Biol 2009; 143: 84-7.

27. MATSHUI H, et al. Gravidez precoce resultados após a quimioterapia para tumor trofoblástico gestacional. J Reprod Med. 2004; 49 (7):531-4.

28. NOBUHARA I, et al. Doença gestacional metastática múltipla trofoblástica depois de uma gestação gemelar com mola. Journal of obstetrics \& Gynecology 57. 2018, $588-593$.

29. OLIVEIRA AS, et al.Gestação gemelar com mola hidatiforme completa e feto vivo após ovodoacão e fertilização em vitro: relato de caso. Reprod clim . 2013; 28 (3): 130-134.

30. PIURA B, et al. Twin com uma mola hidatidiforme completa e sobreviver a co-existente feto. Arch Gynecol Obstet. 2008; 278 (4): 377-382.

31. SEBIRE NJ, et al.Resultado das gestações gemelares com mola hidatiforme completa e co-twin saudável.Lanceta. 2002; 359 (9324): 2165-6.

32. SECKL MJ, et al. trofoblástica gestacional doença. Lanceta. 2010; 376 (9742): 717-29.

33. SOARES LR, et al. Gestação gemelar com mola hidatiforme completa e feto vivo Reprodução e climatério. $2014 ; 29$ (2): 80-83

34. SOARES MKP, et al. Tratamentos na doença trofoblástica gestacional: uma revisão integrativa Revista de Enfermagem do Centro-Oeste Mineiro 2017; 7/1838.

35. SUKSAI M, et al. European Journal of Obstetrics \& Gynecology and Reproductive Biology 212; 2017 1-8.

36. WAGNER SS, et al. Metastático gestacional doença trofoblástica após uma completa mola Hidatiforme mistura com um Anencefálico feto diagnosticado emuma gestação de 10 semanas. J Ultrasound Med 2008; 27: 1533-1536.

37. YELA DA, et al. Gestação gemelar de mola hidatiforme completa com feto vivo. J Bras Patol Med Lab. 2011; 47: 16570.

38. ZILBERMAN SN. É uma mola hidatiforme completa e um feto normal coexistente um efeito iatrogênico. 2019. 